\title{
Evaluation of clinical pharmacist recommendations in the geriatric ward of a Belgian university hospital
}

This article was published in the following Dove Press journal:

Clinical Interventions in Aging

12 June 2013

Number of times this article has been viewed

\author{
Annemie Somers' \\ Hugo Robays' \\ Peter De Paepe ${ }^{2}$ \\ Georges Van Maele ${ }^{3}$ \\ Katrina Perehudoff ${ }^{4}$ \\ Mirko Petrovic ${ }^{4}$ \\ 'Department of Pharmacy, Ghent \\ University Hospital, Ghent, Belgium; \\ ${ }^{2}$ Department of Emergency Medicine, \\ Ghent University Hospital, Ghent, \\ Belgium; ${ }^{3}$ Department of Medical \\ Statistics, Ghent University Hospital, \\ Ghent, Belgium; ${ }^{4}$ Department of \\ Geriatrics, Ghent University Hospital, \\ Ghent, Belgium
}

Objective: To evaluate the type, acceptance rate, and clinical relevance of clinical pharmacist recommendations at the geriatric ward of the Ghent university hospital.

Methods: The clinical pharmacist evaluated drug use during a weekly 2-hour visit for a period of 4 months and, if needed, made recommendations to the prescribing physician. The recommendations were classified according to type, acceptance by the physician, prescribed medication, and underlying drug-related problem. Appropriateness of prescribing was assessed using the Medication Appropriateness Index (MAI) before and after the recommendations were made. Two clinical pharmacologists and two clinical pharmacists independently and retrospectively evaluated the clinical relevance of the recommendations and rated their own acceptance of them.

Results: The clinical pharmacist recommended 304 drug therapy changes for 100 patients taking a total of 1137 drugs. The most common underlying drug-related problems concerned incorrect dose, drug-drug interaction, and adverse drug reaction, which appeared most frequently for cardiovascular drugs, drugs for the central nervous system, and drugs for the gastrointestinal tract. The most common type of recommendation concerned adapting the dose, and stopping or changing a drug. In total, $59.7 \%$ of the recommendations were accepted by the treating physician. The acceptance rate by the evaluators ranged between $92.4 \%$ and $97.0 \%$. The mean clinical relevance of the recommendations was assessed as possibly important (53.4\%), possibly low relevance (38.1\%), and possibly very important (4.2\%). A low interrater agreement concerning clinical relevance between the evaluators was found: kappa values ranged between 0.15 and 0.25. Summated MAI scores significantly improved after the pharmacist recommendations, with mean values decreasing from 9.3 to $6.2(P<0.001)$.

Conclusion: In this study, the clinical pharmacist identified a high number of potential drug-related problems in older patients; however, the acceptance of the pharmacotherapy recommendations by the treating physician was lower than by a panel of evaluators. This panel, however, rated most recommendations as possibly important and as possibly having low relevance, with low interrater reliability. As the appropriateness of prescribing seemed to improve with decreased MAI scores, clinical pharmacy services may contribute to the optimization of drug therapy in older inpatients.

Keywords: clinical pharmacist, drug-related problems, geriatric inpatients

\section{Introduction}

Clinical pharmacy is defined as the provision of patient-oriented pharmaceutical care, with the goals of maximizing drug efficacy and minimizing drug harm by preventing drug-related problems. ${ }^{1}$ In hospitals, clinical pharmacists are working on the wards and are part of the interprofessional team, where they analyze the pharmacotherapy 
of the patients in relation to the medical and laboratory data. They recommend drug therapy changes to physicians and nurses, and they answer questions about drugs.

Clinical pharmacy has been practiced for many decades in North America and the United Kingdom. It was introduced in most European countries several years ago. About 10 years ago, clinical pharmacy pilot projects were started in Belgian university hospitals, and for the past few years the Belgian government has financed clinical pharmacy projects in about 50 hospitals. Many of these projects target older patients, in whom drug consumption is high and the presence of many drug-related problems requires a careful assessment of drug therapy. Clinical pharmacy was introduced at the geriatric ward in our hospital as a result of the high rate of drug-related hospital admissions and drug-related problems during hospitalization., ${ }^{2,3}$

The clinical and economic impact of clinical pharmacy has been addressed in many studies, and the value of clinical pharmacy has been proven through the improvement of both clinical and economic outcomes. ${ }^{4-10}$ This study took place before clinical pharmacy activities were financially supported by the Belgian government. Therefore, it was important to document not only the type of recommendations, but also their acceptance by the treating physicians in our hospital. Moreover, assessing the clinical relevance of the pharmacist recommendations was a key objective in the light of the extension of clinical pharmacy to other departments in the hospital.

\section{Methods}

\section{Setting and study design}

This observational monocentric study was performed at the geriatric ward (28 beds) of the Ghent University Hospital, Belgium, during a 4-month period (April-July 2006). Geriatric patients admitted to this acute geriatric ward were included in the study. Three geriatricians, assisted by two junior physicians, were active on the ward. The geriatricians adhered to a 6-month rotation system for supervision, which was performed by a single geriatrician during the study period. The clinical pharmacist started the activities at the geriatric ward 6 months before the beginning of the study.

\section{Clinical pharmacist activity}

The clinical pharmacist attended the geriatric ward weekly for 2 hours, during which time pharmacotherapy recommendations were formulated according to a standardized working method and after consulting the medical and nursing files and the laboratory values. At the time of the study, the medical file and the laboratory values were available in the electronic patient file, but the nursing file (including the pharmacotherapy chart) was still a paper document. The pharmacist screened the pharmacotherapy charts for eight types of underlying drug-related problems per drug: incorrect dose, inappropriate drug choice, drug-drug interaction, adverse drug reaction, incorrect frequency or time of administration, incorrect route of administration, unnecessary use of the drug, and contraindication. Also, the pharmacist screened for untreated indications. The recommendations were formulated by means of a standardized form, which was inserted in the nursing file next to the pharmacotherapy chart. On the form, the drug-related problem was specified (mostly with a short explanation; eg, the mechanism of a drug-drug interaction), as well as the pharmacotherapy recommendation. The next day, during their rounds, the geriatrician and the junior physician noted on the form whether they accepted the suggestion of the pharmacist. Reasons for not accepting the recommendation were recorded, but were not always noted by the physician. The acceptance rates were coded as "accepted," "partially accepted," and "not accepted." A partially accepted recommendation was coded if, for example, the pharmacist recommended to lower the dose of a drug and the drug was entirely stopped, or if the pharmacist recommended to start a drug and this drug was initiated but at a different dose than proposed. The pharmacist did not attend the ward rounds due to time restrictions, and because many other issues besides drug therapy were discussed during this round. Recommendations were followed up 1 day after the ward round by collecting the forms and by checking whether the pharmacotherapy regimen was altered. In cases where the physician's acceptance decision was not noted or where an accepted recommendation did not lead to a change in pharmacotherapy, the pharmacist contacted the treating physician for clarification. Only the patients for whom recommendations were formulated and only pharmacist-initiated recommendations (not questions) were taken into account.

\section{Panel evaluation}

Four health care professionals independently and retrospectively rated the clinical relevance of the recommendations and assessed their own acceptance of the recommendations. These evaluators were two clinical pharmacologists (one geriatrician and one emergency medicine physician) and two clinical pharmacists with more 
than 3 years of experience each. The clinical relevance of the recommendations was assessed by a previously developed scale, ${ }^{11}$ with six levels of relevance: "not relevant," "possibly low relevance," "possibly important relevance," "possibly very important relevance," "possibly life-saving," and "adverse significance" (in case an inappropriate recommendation was given). Instructions for rating were provided both by a letter and by personalized communication.

\section{Appropriateness of prescribing}

The Medication Appropriateness Index (MAI) scoring system was used to assess the appropriateness of prescribing before and after the pharmacist recommendations were made. ${ }^{12}$ This method has been used frequently for measuring interventions to improve pharmacotherapy in older patients. ${ }^{6,7,13,14}$ In this approach, each drug is evaluated as appropriate, marginally appropriate, or inappropriate by ten weighted criteria, resulting in a score per drug and in a summated score per patient. Inappropriate ratings for criteria with a high weight generate the highest scores. In the original MAI, ten questions are posed per drug taken; these ten questions concern indication, effectiveness, dosage, correct directions, practical directions, drug-drug interactions, drugdisease interactions, duplication, duration, and expense. In our approach, the question about drug expense was not considered, since defining the least expensive drug will differ according to which perspective is taken into account, owing to a complex reimbursement system with fixed drug budget for hospitalized patients. The MAI scores were calculated by a clinical pharmacist and a geriatrician (together), both experienced with MAI ratings. The second MAI score was calculated based on the acceptance of the treating physician; this means that rejected recommendations were not taken into consideration.

\section{Data analysis}

Patient data, drug therapy, and clinical pharmacist recommendations were recorded into an MS Access database (version 2007; Microsoft Corporation, Redmond, WA, USA). Statistical analysis was performed by using SPSS version 19 (SPSS, Inc, Chicago, IL, USA). The interrater variability was assessed by the kappa statistics, whereby raters were compared two by two. A kappa value of $>0.8$ indicates nearly perfect interrater agreement; kappa values between 0.6 and 0.8 indicate substantial agreement, and kappa values between 0.4 and 0.6 indicate moderate agreement. The difference in MAI scores before and after the recommendation was calculated by the Wilcoxon matched-pairs signed-rank test. A $P$-value $<0.05$ was considered statistically significant.

\section{Results Clinical pharmacist recommendations}

The clinical pharmacist formulated pharmacotherapy recommendations for 100 patients ( 52 males and 48 females) with a mean age of 81.4 years (standard deviation [SD], 6.65 ; median, 82 years). Twenty-three patients came from a nursing home or from another hospital, and four patients died during the hospital stay. The 100 patients took a total of 1137 drugs (mean 11.4 drugs, SD 3.36; median, 11 drugs; range, 4-23), which were mainly cardiovascular drugs (25.4\%), central nervous system drugs $(21.8 \%)$, drugs for the gastrointestinal tract $(20.2 \%)$, and drugs for blood disorders (11.1\%).

In total, the clinical pharmacist recommended 304 drug therapy changes, with a maximum of six recommendations per patient. Table 1 summarizes the most common underlying potential drug-related problems, which most frequently concerned inappropriate dose (31\%), drug-drug interaction $(20 \%)$, and adverse drug reaction (15\%). The most common recommendation types were changing the dose (35\%), stopping a drug (18\%), changing a drug (14\%), and changing the pharmaceutical form (9\%). Examples of recommendations are given in Table 2. The pharmacotherapeutic drug classes most frequently involved in drug-related problems were cardiovascular drugs $(32 \%)$, central nervous system drugs $(26 \%)$, drugs for the gastrointestinal tract $(11 \%)$, and antiinfectives $(10 \%)$.

\section{Acceptance rates}

Table 3 illustrates the acceptance rates by the treating physicians and by the four evaluators. The treating physicians (the supervising geriatrician and the junior physicians) accepted 53.6\% and partially accepted $6.3 \%$ of the recommendations. The most frequently accepted types of recommendations were monitoring therapy (93.8\% accepted), changing the pharmaceutical form $(85.7 \%)$, and advice to measure the drug concentration (84.6\%), as well as to stop the drug (69.8\%); the least often accepted types of recommendations were changing the drug $(62.5 \%$ not accepted), starting a drug (60.9\%), and changing the dose (49.5\%). Two of the 304 recommendations were not evaluated by all four evaluators due to a lack of clinical information. The four evaluators fully accepted $86.1 \%, 87.4 \%, 86.8 \%$, and $79.8 \%$ of the recommendations, respectively, and partially accepted $7.0 \%, 9.6 \%, 7.0 \%$, and $12.6 \%$, respectively. 
Table I Characteristics of underlying drug-related problems $(n=304)$

\begin{tabular}{|c|c|c|}
\hline Problem & Number (\%) & Drug type \\
\hline Inappropriate dose & 95 (31\%) & $\begin{array}{l}\text { Statins, proton pump inhibitors, anti-infectives, hypnotics, ACE } \\
\text { inhibitors, antithrombotics }\end{array}$ \\
\hline Drug-drug interaction & $62(20 \%)$ & Antithrombotics, digoxin, anti-infectives, psycholeptics, psychoanaleptics \\
\hline Adverse effect & $46(15 \%)$ & Diuretics, ACE inhibitors, corticosteroids, psycho(ana)leptics \\
\hline Inadequate form & $32(11 \%)$ & Analgesics, psycholeptics \\
\hline Unnecessary drug & $22(7 \%)$ & Miscellaneous \\
\hline Wrong drug choice & $22(7 \%)$ & Cardiovascular drugs (beta blockers, diuretics, digoxin, ACE inhibitors) \\
\hline $\begin{array}{l}\text { Inappropriate frequency or } \\
\text { administration time }\end{array}$ & $9(3 \%)$ & Anti-infectives, calcium/vitamin D, statins \\
\hline Underuse & $8(3 \%)$ & Calcium/vitamin D \\
\hline Contraindication & $5(2 \%)$ & NSAIDs, antidiabetic drugs, antibiotics, beta blockers \\
\hline Inappropriate route of administration & $2(1 \%)$ & Anti-infectives \\
\hline
\end{tabular}

Abbreviations: ACE, angiotensin-converting enzyme; NSAID, nonsteroidal anti-inflammatory drug.

\section{Clinical relevance}

The clinical relevance of the recommendations (Table 4) was mostly scored as "possibly important" (mean, 53.4\%) and "possibly low relevance" (mean, 38.1\%). The assessment "possibly very important" was not attributed by all evaluators (mean, 4.2\%). No recommendations were designated as being of possible life-saving clinical relevance. One recommendation $(0.3 \%)$ was categorized as having adverse significance by all the evaluators, since the pharmacist had made a mistake by suggesting treatment for hyperkalemia instead of hypokalemia.
All kappa values were low (between 0.25 and 0.15 ), as well as those between the two clinical pharmacologists and those between the two clinical pharmacists, indicating a poor agreement between the raters.

\section{MAl scores}

The mean summated MAI scores decreased from 9.3 (median, 8; SD, 5.12; range, 2.0-36.5) before the recommendation to 6.2 (median, 6; SD, 4.00; range, 0.0-26.5) after the recommendation. This decrease was significant $(P<0.001)$.

Table 2 Examples of clinical pharmacist recommendations

\begin{tabular}{|c|c|c|c|}
\hline Problem & Drug & Recommendation & $\begin{array}{l}\text { Physician } \\
\text { decision }\end{array}$ \\
\hline \multicolumn{4}{|c|}{ Recommendations assessed as having possibly moderate clinical relevance (by all four evaluators) } \\
\hline Unnecessary drug & Ranitidine 300 mg QD & $\begin{array}{l}\text { Stop drug; no concomitant drugs with corrosive effect on the } \\
\text { gastric mucosa. (Reason for hospital admission not related to } \\
\text { gastrointestinal pathology). }\end{array}$ & Accepted \\
\hline Inappropriate dose & Simvastatin $40 \mathrm{mg}$ QD & $\begin{array}{l}\text { Lower dose to } 20 \mathrm{mg} \text { QD since cholesterol levels were } \\
\text { excellent (TC I I } 3 \mathrm{mg} / \mathrm{dL} \text {, LDL } 53 \mathrm{mg} / \mathrm{dL} \text { ), for woman } \\
87 \text { years old; no instable angina, no diabetes. }\end{array}$ & Not accepted \\
\hline \multicolumn{4}{|c|}{ Recommendations assessed as having possibly important clinical relevance (by all four evaluators) } \\
\hline Drug-drug interaction & Haloperidol 5 drops TID & $\begin{array}{l}\text { Stop drug due to interaction with amiodarone and } \\
\text { domperidone: increased risk of QT prolongation; consider } \\
\text { restarting after domperidone therapy as needed for agitation. }\end{array}$ & Accepted \\
\hline Wrong drug choice & Carvedilol 12.5 mg QD & $\begin{array}{l}\text { Patient with heart failure, ischemic heart disease, diabetes, } \\
\text { and COPD receiving two beta blockers (nebivolol and } \\
\text { carvedilol) but no ACE inhibitor; stop carvedilol (nonselective) } \\
\text { and start lisinopril } 10 \mathrm{mg} \text { QD. }\end{array}$ & Not accepted \\
\hline \multicolumn{4}{|c|}{ Recommendations assessed as having possibly very important clinical relevance (by two evaluators) } \\
\hline Drug underuse & Calcium + vitamin D & $\begin{array}{l}\text { Start calcium + vitamin D (and treatment with alendronate } \\
70 \text { mg weekly) for } 83 \text {-year-old woman with known osteoporosis } \\
\text { treated with methylprednisolone for several weeks. }\end{array}$ & Accepted \\
\hline Contraindication & Nitrofurantoin 100 mg BID & $\begin{array}{l}\text { Switch to other antibiotic (eg, levofloxacin } 500 \mathrm{mg} \text { initially, then } \\
250 \mathrm{mg} \text { QD) because of decreased renal function (calculated } \\
\text { GFR: } 35 \mathrm{~mL} / \text { minute). }\end{array}$ & Accepted \\
\hline
\end{tabular}

Abbreviations: QD, once a day; TC, total cholesterol; LDL, low-density lipoprotein; TID, three times a day; COPD, chronic obstructive pulmonary disease; ACE, angiotensin-converting enzyme inhibitor; BID, twice a day; GFR, glomerular filtration rate. 
Table 3 Acceptance rates of clinical pharmacist recommendations $(n=302)$

\begin{tabular}{|c|c|c|c|c|c|}
\hline & \multirow[t]{2}{*}{$\begin{array}{l}\text { Treating } \\
\text { physician }\end{array}$} & \multicolumn{2}{|c|}{$\begin{array}{l}\text { Physician/clinical } \\
\text { pharmacologists }\end{array}$} & \multicolumn{2}{|c|}{$\begin{array}{l}\text { Clinical } \\
\text { pharmacists }\end{array}$} \\
\hline & & Rater I & Rater 2 & Rater 3 & Rater 4 \\
\hline Accepted & $53.6 \%$ & $86.1 \%$ & $87.4 \%$ & $86.8 \%$ & $79.8 \%$ \\
\hline $\begin{array}{l}\text { Partially } \\
\text { accepted }\end{array}$ & $6.3 \%$ & $7.0 \%$ & $9.6 \%$ & $7.0 \%$ & $12.6 \%$ \\
\hline Not accepted & $40.1 \%$ & $7.0 \%$ & $3.0 \%$ & $6.3 \%$ & $7.6 \%$ \\
\hline
\end{tabular}

Abbreviation: $\mathrm{n}$, number.

The criteria with the highest (most inappropriate) scores were drug-drug interactions, dosage, and drug choice, both for the calculation before and after the recommendation. The lowest scores were found for duplication of therapy, indication, and drug-disease interactions. All mean scores per criterion decreased after the pharmacist recommendations. The scores for drug-drug interactions and dosage obtained the highest improvement (decrease from 2.65 to 1.8 and from 2.05 to 1.22 , respectively).

\section{Discussion}

\section{Clinical pharmacist recommendations}

In this study, we evaluated the type, acceptance rate, and clinical relevance of clinical pharmacist recommendations at the geriatric ward of the Ghent University Hospital. The clinical pharmacist focused on the analysis of pharmacotherapy during hospitalization, not on medication history upon admission and not on information at discharge, meaning that only the inpatient drug therapy was analyzed. The acceptance rate of the clinical pharmacist recommendations was somewhat lower than what has been reported in other studies. ${ }^{9}, 13,15-17$ This could be explained by the fact that the pharmacist was not present when the recommendations were considered by the treating physician, precluding further discussion at that time. By spending more time at the geriatric ward, the clinical pharmacist could become better integrated in the multidisciplinary team, which may enhance the team's awareness of drug-related problems.
This study found that the acceptance rate of the four evaluators was higher than that of the treating physicians. The reasons for rejection were mostly found in the notes and could be discussed later with the treating physicians. Three main reasons for not accepting the clinical pharmacist recommendations were identified: (1) the recommendation, even if valuable according to evidence-based medicine, was not taken into account because of the already precarious situation of the patient (eg, the patient was very ill; the physician decided not to change drug therapy because in this case the benefits outweighed the possible risk); (2) the recommendation was not valuable enough according to the treating physician (eg, dose reduction of an antibiotic or digoxin was not taken into account since there were no visible signs of toxicity); and (3) the drug was initially started by another physician (eg, a cardiologist or the general practitioner), and the treating physician did not want to interfere. It is clear that the four evaluators did not take the second and the third motivation into account, since these are overruled by a comprehensive pharmacotherapy approach (eg, as mentioned in criteria for inappropriate prescribing in the elderly, ${ }^{18}$ the dose of digoxin should not exceed $0.125 \mathrm{mg}$ in elderly patients in view of the increased risk of toxicity, even if serum concentration levels are below the maximum value). However, the first motivation is valuable and can only be judged by the treating physician.

\section{Clinical relevance}

The majority of the recommendations were considered as having an important or low clinical relevance, demonstrating that the clinical pharmacist was able to detect potential drugrelated problems and to give adequate recommendations. However, little agreement was found between the raters, including not between the two physicians or between the two pharmacists. This could be due to the fact that the raters were not experienced at evaluating pharmacotherapy recommendations and worked entirely independently of one another. This situation could have been improved by piloting the assessment on test cases in a preliminary test phase

Table 4 Clinical relevance of clinical pharmacist recommendations $(n=302)$

\begin{tabular}{|c|c|c|c|c|c|}
\hline & Rater I & Rater 2 & Rater 3 & Rater 4 & Mean \\
\hline 0 - Adverse significance & $0.3 \%$ & $0.3 \%$ & $0.3 \%$ & $0.3 \%$ & $0.3 \%$ \\
\hline I - No clinical relevance & $1.0 \%$ & $1.7 \%$ & $6.3 \%$ & $7.0 \%$ & $4.0 \%$ \\
\hline 2 - Possibly low relevance & $40.1 \%$ & $24.8 \%$ & $43.7 \%$ & $43.7 \%$ & $38.1 \%$ \\
\hline 3 - Possibly important relevance & $58.6 \%$ & $68.5 \%$ & $43.4 \%$ & $43.0 \%$ & $53.4 \%$ \\
\hline 4 - Possibly very important relevance & $0.0 \%$ & $4.6 \%$ & $6.3 \%$ & $6.0 \%$ & $4.2 \%$ \\
\hline 5 - Possibly life-saving & $0.0 \%$ & $0.0 \%$ & $0.0 \%$ & $0.0 \%$ & $0.0 \%$ \\
\hline
\end{tabular}

Abbreviation: $\mathrm{n}$, number. 
followed by a meeting of the four raters to discuss the cases and to streamline the rating process. Similar low agreement rates were found in other studies, suggesting the difficulty in assessing this type of intervention. ${ }^{13,19}$

Another measure of clinical relevance was the decrease in MAI score. Although one aspect (expense) was not considered in our study, this finding is consistent with the findings of another study in Belgium, where MAI scores decreased in a randomized controlled trial of clinical pharmacist's interventions for geriatric patients. ${ }^{7}$

\section{Strengths and weaknesses}

This study was observational and monocentric, and only one clinical pharmacist was involved. Therefore, the results should be extrapolated with caution. The patient characteristics and the types of drugs prescribed at the acute geriatric ward are probably comparable to other hospitals; however, the prescribing rules and the working method of the physicians and pharmacists may differ between institutions. Also, the detection of drug-related problems and the recommendations that were provided could strongly depend on the experience of the clinical pharmacist involved. The pharmacist in this study benefited from extensive training in geriatric pharmacotherapy and used a systematic and evidence-based approach for the detection of drug-related problems.

No clinical endpoints were taken into consideration (eg, hospital readmissions, mortality, and quality of life). Also, the lack of a control group of patients not undergoing the intervention can be considered a weakness of the study design. A randomized controlled design with an analysis of the morbidity and mortality outcomes would have been preferable. However, it should be clear that studying this type of intervention is rather difficult because the presence of the pharmacist alone can influence the pharmacotherapy, and the pharmacist recommendations in one case can influence the pharmacotherapy of other patients. As a result of the limited time investment of the clinical pharmacist (only 2 hours per week), such a design was not possible. Moreover, the aim of this study was not to assess the impact of the clinical pharmacist on clinical outcomes. Instead, the goal was to evaluate the characteristics of the clinical pharmacist recommendations and whether these seemed relevant according to a panel of experts in pharmacotherapy.

The strengths of this study lie in the relatively high number of patients that were included and the quantity of recommendations that had been given in the 4-month study period, in spite of the clinical pharmacist's limited availability. Many other recommendations to improve therapy were given, although they are outside the scope of this study because they are now covered by the electronic prescribing system in our hospital. Examples include the clarification of prescriptions and drug charts (eg, where doses were not mentioned), and recommendations concerning pharmacotechnical aspects (eg, how to administer antibiotic infusions). Furthermore, the provision of drug information outside of any recommendation (eg, a warning for a drugdrug interaction when neither of the two drugs could be changed) was not included in the analysis. Another possible strength is the structured approach, whereby the clinical pharmacist used a list of potential drug-related problems, and where the MAI scores were calculated.

\section{Conclusion}

This study demonstrates that by working in a systematic way, a clinical pharmacist was able to detect a high number of drug-related problems in older patients in a limited amount of time. Therefore, it seems that the role of the clinical pharmacist for older patients might be to detect the over- and underuse of drugs, and to discuss therapeutic goals and drug use with physicians. Our results also suggest that the role of a clinical pharmacist for geriatric inpatients should be enlarged, since the majority of the recommendations were rated as possibly important, and since the appropriateness of prescribing improved under the guidance of a clinical pharmacist.

\section{Acknowledgments}

The authors are indebted to the participating geriatricians, professors Dr N Van Den Noortgate, Dr A Velghe, Dr P De Smet, and Dr K De Wilde; to head nurse, $H$ Van Doninck; and to the patients of the geriatric ward. Furthermore, we thank the clinical pharmacists (S Deryckere and S Mertens) for their evaluation of the recommendations. Useful comments for this manuscript were provided by Professor Dr M Bogaert. This study was not funded.

\section{Disclosure}

The authors report no conflicts of interest in this work.

\section{References}

1. Hepler CD, Strand LM. Opportunities and responsibilities in pharmaceutical care. Am J Hosp Pharm. 1990;47(3):533-543.

2. Somers A, Petrovic M, Robays H, Bogaert M. Reporting adverse drug reactions on a geriatric ward: a pilot project. Eur J Clin Pharmacol. 2003;58(10):707-714.

3. Somers A, Robays H, Vander Stichele R, Van Maele G, Bogaert M, Petrovic M. Contribution of drug related problems to hospital admission in the elderly. J Nutr Health Aging. 2010;14(6):477-482. 
4. Bond CA, Raehl CL, Franke T. Interrelationships among mortality rates, drug costs, total cost of care, and length of stay in United States hospitals: summary and recommendations for clinical pharmacy services and staffing. Pharmacotherapy. 2001;21(2):129-141.

5. Brown BK, Earnhart J. Pharmacists and their effectiveness in ensuring the appropriateness of the chronic medication regimens of geriatric inpatients. Consult Pharm. 2004;19(5):432-436.

6. Gillespie U, Alassaad A, Henrohn D, et al. A comprehensive pharmacist intervention to reduce morbidity in patients 80 years or older: a randomized controlled trial. Arch Intern Med. 2009;169(9):894-900.

7. Spinewine A, Swine C, Dhillon S, et al. Effect of a collaborative approach on the quality of prescribing for geriatric inpatients: a randomized, controlled trial. J Am Geriatr Soc. 2007;55(5):658-665.

8. Kaboli PJ, Hoth AB, McClimon BJ, Schnipper JL. Clinical pharmacists and inpatient medical care: a systematic review. Arch Intern Med. 2006; 166(9):955-964.

9. Kucukarslan SN, Peters M, Mlynarek M, Nafziger DA. Pharmacists on rounding teams reduce preventable adverse drug events in hospital general medicine units. Arch Intern Med. 2003;163(17):2014-2018.

10. Holland R, Lenaghan E, Harvey I, et al. Does home based medication review keep older people out of hospital? The HOMER randomised controlled trial. BMJ. 2005;330(7486):293.

11. Overhage JM, Lukes A. Practical, reliable, comprehensive method for characterizing pharmacists' clinical activities. Am J Health Syst Pharm. 1999;56(23):2444-2450
12. Hanlon JT, Schmader KE, Samsa GP, et al. A method for assessing drug therapy appropriateness. J Clin Epidemiol. 1992;45(10):1045-1051.

13. Stuijt CC, Franssen EJ, Egberts AC, Hudson SA. Appropriateness of prescribing among elderly patients in a Dutch residential home: observational study of outcomes after a pharmacist-led medication review. Drugs Aging. 2008;25(11):947-954.

14. Hanlon JT, Artz MB, Pieper CF, et al. Inappropriate medication use among frail elderly inpatients. Ann Pharmacother. 2004;38(1):9-14.

15. Spinewine A, Dhillon S, Mallet L, Tulkens PM, Wilmotte L, Swine C. Implementation of ward-based clinical pharmacy services in Belgium - description of the impact on a geriatric unit. Ann Pharmacother. 2006;40(4):720-728.

16. Bosma L, Jansman FG, Franken AM, Harting JW, Van den Bernt PM. Evaluation of pharmacist clinical interventions in a Dutch hospital setting. Pharm World Sci. 2008;30(1):31-38.

17. Leape LL, Cullen DJ, Clapp MD, et al. Pharmacist participation on physician rounds and adverse drug events in the intensive care unit. JAMA. 1999;282(3):267-270.

18. Page RL 2nd, Linnebur SA, Bryant LL, Ruscin JM. Inappropriate prescribing in the hospitalized elderly patient: defining the problem, evaluation tools, and possible solutions. Clin Interv Aging. 2010;5:75-87.

19. Buurma H, De Smet PA, Leufkens HG, Egberts AC. Evaluation of the clinical value of pharmacists' modifications of prescription errors. $\mathrm{Br}$ J Clin Pharmacol. 2004;58(5):503-511.
Clinical Interventions in Aging

\section{Publish your work in this journal}

Clinical Interventions in Aging is an international, peer-reviewed journal focusing on evidence-based reports on the value or lack thereof of treatments intended to prevent or delay the onset of maladaptive correlates of aging in human beings. This journal is indexed on PubMed Central, MedLine, the American Chemical Society's 'Chemical Abstracts

\section{Dovepress}

Service' (CAS), Scopus and the Elsevier Bibliographic databases. The manuscript management system is completely online and includes a very quick and fair peer-review system, which is all easy to use. Visit http://www.dovepress.com/testimonials.php to read real quotes from published authors. 